$x-610 \cdot 64-202$

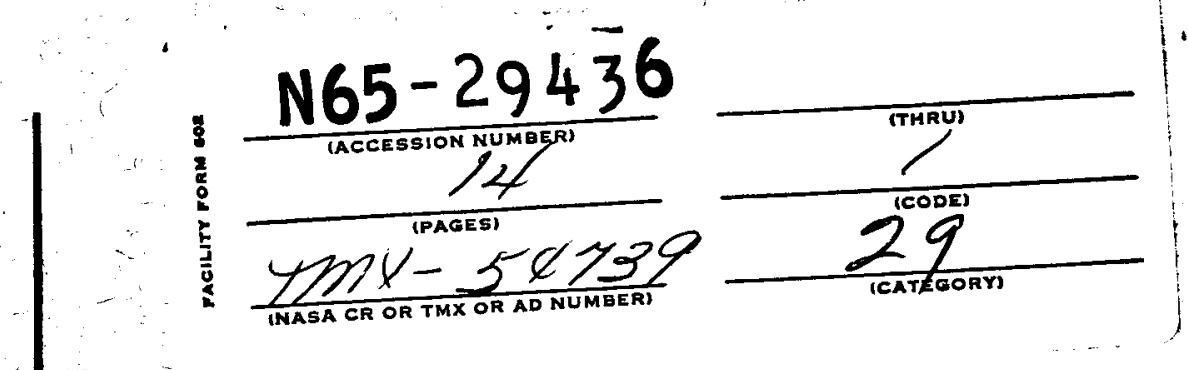

THE UV LINES OF FEXIV IN THE SOLAR CORONA

BY

R. STOCKHAUSEN

GPO PRICE

CFSTI PRICES) \$

Hard copy $(\mathrm{HC}) \frac{1.00}{-50}$

JULY 1964

ff 653 July 65

GODDARD SPACE FLIGHT CENTER

GREENBELT, MARYLAND 


\title{
THE UV LINES OF FE XIV IN THE SOLAR CORONA
}

By

\author{
R. Stockhausen \\ Goddard Space Flight Center \\ Greenbelt, Maryland
}

Knowledge of the structure of the solar corona can be increased by observations of the many emission lines in the far ultraviolet. However, identification of these lines is made difficult by the lack of knowledge of many of the atomic parameters relevant to these lines. Garstang's (1962) calculations of energy levels and oscillator strengths for Fe XIV allow predictions of lines strengths and wave lengths for the lines of this ion.

Collision cross-sections of the optically permitted lines are related to the oscillator strengths by the expression given by Van Regemorter (1962)

$$
\mathrm{Q}(\mathrm{n}, \mathrm{m})=\frac{1}{\mathrm{k}_{\mathrm{n}}{ }^{2}} \frac{8 \pi}{\sqrt{3}} \frac{\mathrm{I}_{\mathrm{H}}}{\Delta \mathrm{E}} \mathrm{f}(\mathrm{nm}) \overline{\mathrm{g}} \pi \mathrm{a}_{\mathrm{o}}{ }^{2}
$$

For levels not connected to the ground level by an optically allowed transition, electron exchange collisions are the only excitation mechanism. In a manner similar to Osterbrock (1963) 
these exchange cross-sections can be estimated by

$$
\begin{aligned}
Q_{e}(i n, j m)<Q_{0}(i n, j m)= & \frac{\pi}{k_{n}^{2}}\left\{\begin{array}{l}
(2 l+1)\left[g_{j m} / \sum_{m j m} g_{j m}\right] \\
(2 l+41)\left[g_{j m} / \sum_{m} g_{j m}\right]\left(g_{j} / g_{j}\right)
\end{array}\right. \\
& \text { or } \frac{\pi}{k_{n}^{2}}\left\{\begin{array}{l}
(2 l+1) \\
\left(2 l^{\prime}+1\right)\left(g_{j m} / g_{i n}\right)
\end{array}\right.
\end{aligned}
$$

whichever of the four is smaller. Here $\underline{i} \underline{n}$ is the $\underline{n}^{\text {th }}$ level of term $\underline{i}$ and $\underline{j} \underline{m}$ is the $\underline{m}^{\text {th }}$ level of term $\underline{j}$. The term in square brackets comes from the assumption that the levels of term $j$ are populated in proportion to this weight factor.

In his work with nebular ions, Osterbrock has taken $Q_{e}=0.1 Q_{0}$. On the basis of the rapid decrease, with increasing energy of the colliding electron, of the cross-sections for exchange collisions in He I (St. John, Miller, and Lin 1964) and the decrease of exchange cross-sections along the iso-electronic sequences shown in Table VIII of Seaton (1958), Dr. Osterbrock has suggested that a value of $Q_{e}$ nearer to $Q_{e}=0.01 Q_{0}$ may be more appropriate for $\mathrm{Fe}$ XIV. With the uncertainties involved, parallel calculations were made under three assumptions :

$$
Q_{e}=\left\{\begin{array}{l}
.0 \\
.1 Q_{0} \\
.01 Q_{0}
\end{array}\right.
$$

The total cross-section is then the sum of (1) and (3).

Having the collision cross-sections, production rates of the UV lines can be calculated. Following the notation of Seaton (1963), 
the rate of excitation from level $\underline{i}$ to level $j$ followed by spontaneous transition to level $\underline{\mathrm{k}}$ is given by

$$
\alpha_{i k}=q_{i j} C_{j k}
$$

where $q_{i j}$ is the collisional excitation rate of $j$ from $\underline{i}$ and $C_{j k}$ is the probability of going to $\underline{k}$ from $j$. For the UV lines of Fe XIV, $C_{j k}$ does not involve collisional de-excitation due to the high spontaneous transition probabilities, and the low coronal electron densities.

Table 1 gives the production rates for UV lines due to transitions ending in the two levels ( 1 denotes ${ }^{2} \mathrm{P}_{1 / 2}^{\circ}$ and 2 denotes $2 \mathrm{P}^{\circ} 3 / 2$ ) of the ground term. Results are given for two temperatures and three values of the exchange cross-section. Excitations are assumed to take place only from the ground level.

The sum of the production rates to the upper level $\left({ }^{2} \mathrm{P}_{3 / 2}^{\circ}\right)$ of the ground term is important in that it gives the excitation rate (due to cascade) of the coronal green line, $\lambda 5303$. It can be seen that there is a slight discrepancy between these cascade excitation rates and those given in Table 6 of Seaton (1963). In a private communication, $\mathrm{Dr}$. Seaton has noted a numerical error in his work.

Inclusion of the exchange collisions has a significant effect on the production rates only for those levels which have low $f$ values with respect to the ground level, and then only for the case $Q_{e}=0.1 Q_{0}$. 
TABLE 1

\section{PRODUCTION RATES}

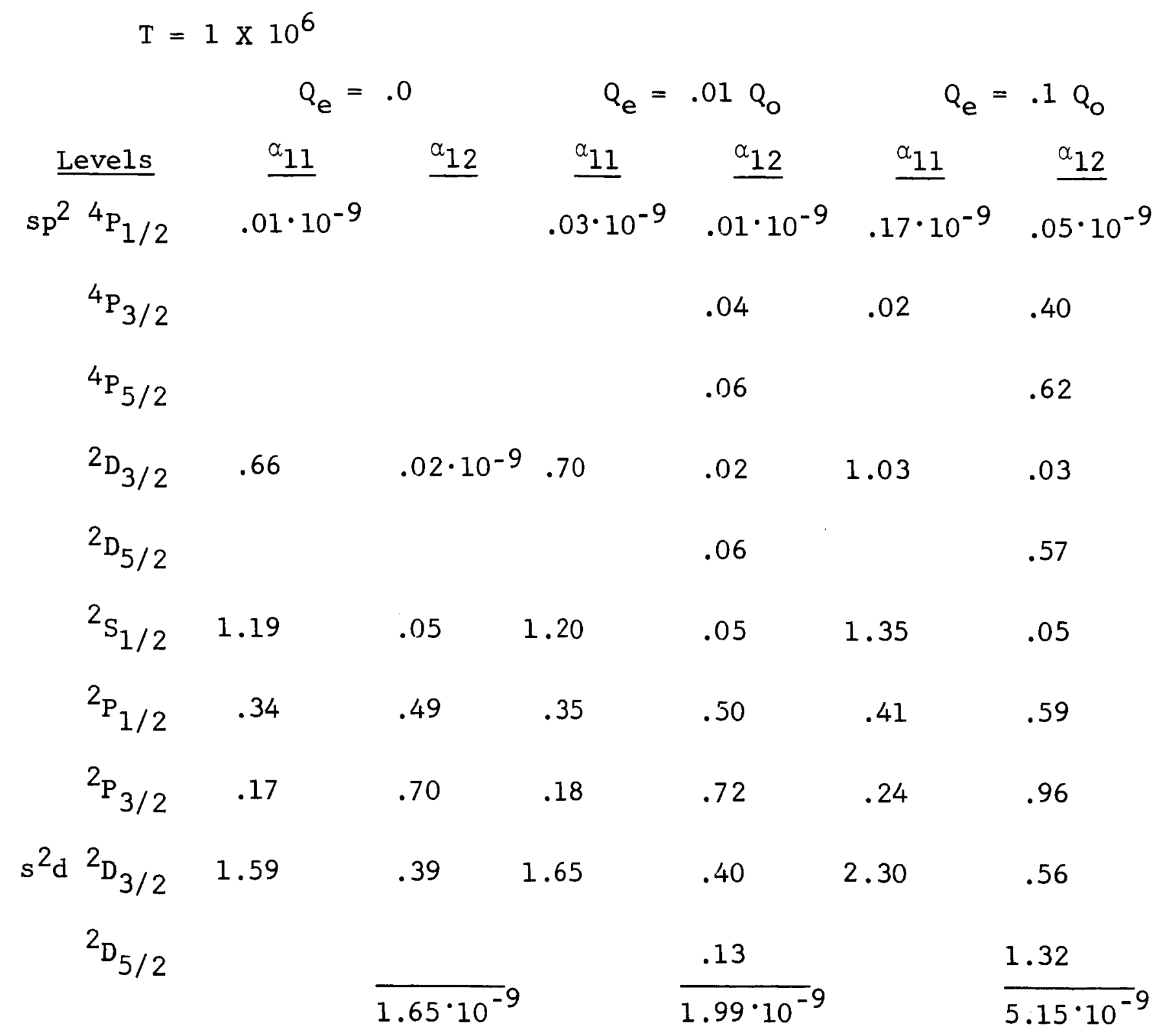




\section{TABLE 1 (Continued)}

\section{PRODUCTION RATES}

$$
\begin{aligned}
& T=2 \times 10^{6} \\
& \mathrm{Q}_{\mathrm{e}}=.0 \\
& \mathrm{Q}_{\mathrm{e}}=.01 \mathrm{Q}_{\mathrm{o}} \\
& \mathrm{Q}_{\mathrm{e}}=.1 \mathrm{Q}_{\mathrm{o}}
\end{aligned}
$$

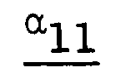


The product of these production rates and the energy of the respective emitted photons, normalized so that the ${ }^{2} \mathrm{P}_{1 / 2}^{\circ}-{ }^{2} \mathrm{P}_{3 / 2}$ transition is unity, gives the predicted relative line strengths of the expected UV lines. These strengths are given in Table 2 . Table 3 gives the predicted wavelengths and the wavelengths assigned by Neupert and Smith (1964) from observations of the NASA satellite OSO-I. These identifications were made on the basis of time variations of the line strengths and wavelength separations of the multiplet members. Also included are the observed relative line strengths for two periods of observation in 1962. Quiet Sun (average of March $8,9,10$ ) is a period of low solar activity and April 20 is a period of high solar activity. These observed line strengths are also normalized to the ${ }^{2} \mathrm{P}_{1 / 2}{ }^{-}{ }^{2} \mathrm{P}_{3 / 2}$ line. This line was about 1.5 times more intense on April 20 than during the Quiet Sun period. At this stage of the reductions, the observed relative line strengths are accurate to only a few tenths (in these units).

In comparing the observations and predictions, it must be noted that the satellite observations do not cover the spectral region occupied by the ${ }^{2} \mathrm{P}^{\circ}-{ }^{4} \mathrm{P}$ multiplet. For the $\mathrm{s}^{2} \mathrm{P}\left({ }^{2} \mathrm{P}^{\circ}\right)-\mathrm{sp}^{2}\left({ }^{2} \mathrm{D}\right)$ multiplet, no lines of the predicted strengths or with the correct time behavior appear to be present at the predicted wavelengths, however the instrumental sensitivity is decreasing in this region. Since these observed strengths are a result of contributions from the entire solar disc, and due to the uncertainties in the 
PREDICTED LINE STRENGTHS

Levels $\mathrm{J}_{\mathrm{L}} \quad \mathrm{J}_{\mathrm{U}} \quad \underline{I}$

$$
\mathrm{T}=1 \times 10^{6} \quad \mathrm{~T}=2 \times 10^{6}
$$

$3 s^{2} \mathrm{p}-3 \mathrm{sp} \mathrm{p}^{2}$

$$
Q_{e}=.0 \quad Q_{e}=.01 Q_{0} \quad Q_{e}=.1 Q_{0}
$$$$
Q_{e}=.0
$$

$Q_{e}=.01 Q_{0}$

.5

.3

$1 / 2 \quad 1 / 2$

$3 / 2 \quad 1 / 2$

$3 / 2 \quad 3 / 2$

$3 / 2 \quad 5 / 2$

${ }^{2} \mathrm{P}^{\circ-2} \mathrm{D}$

$1 / 2 \quad 3 / 2$

2.8

$3 / 2 \quad 3 / 2$

.1

$3 / 2 \quad 5 / 2$

$\begin{array}{llll}1 / 2 & 1 / 2 & 6.4 & 6.1\end{array}$

$3 / 2 \quad 1 / 2$

$\begin{array}{llll}1 / 2 & 1 / 2 & 2.0 & 1.9\end{array}$

$\begin{array}{llll}1 / 2 & 3 / 2 & 1.0 & 1.0\end{array}$

$\begin{array}{llll}3 / 2 & 1 / 2 & 2.7 & 2.6\end{array}$

$\begin{array}{llll}3 / 2 & 3 / 2 & 3.9 & 3.8\end{array}$

$3 s^{2} p-3 s^{2} d$

$2 \mathrm{P}^{\circ}-2 \mathrm{D}$
.1

.1

.2

2.8

.1

.2

1.6

5.2

.2

1.7

1.0

2.3

3.8

2.5

.1

.1

.1

6.3

6.1

.2

.2

1.9

1.9

1.0

1.0

2.6

2.6

$3.9 \quad 3.8$

.1

1

.1

1.1

3.2

.1

1.4

6.1

.2

2.2

3.0

3.9

$11.2 \quad 10.8 \quad 10.7$

12.8

2.6

$2.5 \quad 2.5$

3.0

6.2

.6

5.9
1.0 $\begin{array}{llll}1 / 2 & 3 / 2 & 10.9 & 10.7\end{array}$

$3 / 2 \quad 3 / 2 \quad 2.6$

$3 / 2 \quad 5 / 2$ 
TABLE 3

OBSERVED RELATIVE INTENSITIES AND WAVELENGTHS

\begin{tabular}{|c|c|c|c|c|c|c|}
\hline$\underline{\text { Levels }}$ & $\underline{J_{L}}$ & $\underline{\mathrm{J}_{\mathrm{U}}}$ & $\lambda_{\underline{P}}$ & $\underline{\lambda_{0}}$ & $\underline{I}$ & \\
\hline $3 s^{2} p-3 s p^{2}$ & & & & & Quiet Sun & April 20 \\
\hline${ }^{2} \mathrm{P}^{\circ} \quad{ }^{2} \mathrm{~S}$ & $1 / 2$ & $1 / 2$ & 272.9 & 274.2 & 1.1 & 1.9 \\
\hline & $3 / 2$ & $1 / 2$ & 287.7 & 288.6 & .6 & .6 \\
\hline${ }^{2} \mathrm{P}^{0} \quad{ }^{2} \mathrm{P}$ & $1 / 2$ & $1 / 2$ & 256.0 & Blend & & \\
\hline & $1 / 2$ & $3 / 2$ & 250.5 & 251.8 & 1.0 & 1.0 \\
\hline & $3 / 2$ & $1 / 2$ & 269.0 & 270.5 & .7 & 1.0 \\
\hline $3 s^{2} p-3 s^{2} d$ & $3 / 2$ & $3 / 2$ & 262.9 & 264.5 & 1.2 & 1.5 \\
\hline${ }^{2} \mathrm{P}^{\circ}$ & $1 / 2$ & $3 / 2$ & 214.1 & 203.9 & .9 & 1.3 \\
\hline & $3 / 2$ & $3 / 2$ & 223.17 & דיר & & \\
\hline & $3 / 2$ & $5 / 2$ & 222.0 & 211.5 & 1.3 & 1.8 \\
\hline
\end{tabular}


data, only semi-quantitative comparisons with the calculated strengths will be made. Most apparent is the relatively large spread in predicted strengths as compared to the observed strengths. Using a temperature of $5 \times 10^{5}$ does not significantly reduce this spread. Exchange collisions do not seem important for most cases. However, for the $s^{2} \mathrm{p}\left({ }^{2} \mathrm{P}^{\circ}\right)-s^{2} \mathrm{~d}\left({ }^{2} \mathrm{D}\right)$ multiplet the ratio of the ratio of the sum of the predicted intensities of the $3 / 2-3 / 2$ and $3 / 2-5 / 2$ lines to the $1 / 2-3 / 2$ line (ratio $=8.8: 11.2$ for $\mathrm{T}=10^{6}$ and $\mathrm{Q}_{e}=0.1 \mathrm{Q}_{0}$ ) is closer (but still in the wrong sense) than for the case where $Q_{e}=0$.

One possibility for the discrepancies in relative strengths is some kind of self-absorption. Due to the very low rate of collisional de-excitation (relative to spontaneous transitions), "true" absorption is not to be expected. Following another suggestion of Dr. Osterbrock, there is a mechanism that seems capable of reducing the spread in predicted relative strengths.

For simplicity consider the ${ }^{2} \mathrm{P}^{\circ}-{ }^{2} \mathrm{~S}$ multiplet. The $1 / 2-1 / 2$ line is predicted to be about 20 or 30 times more intense than the $3 / 2-1 / 2$ line. Now the $1 / 2-1 / 2$ photon can be absorbed by another Fe XIV ion. This ion now has the choice of re-emitting the $1 / 2-1 / 2$ photon or emitting a $3 / 2-1 / 2$ photon. With each absorption, the probability of emitting a $3 / 2$ - $1 / 2$ photon increases, thereby making the relative strengths of the lines more nearly equal. A crude calculation will show that such a mechanism seems reasonable for the corona. 
Considering the photons (resulting from collisional excitation) to be injected into a non-emitting layer of gas, the modified production rates for this multiplet can be expressed as

$$
\begin{aligned}
& \alpha^{\prime}{ }_{11}=q_{1 j} c_{j 1} P_{j 1} \\
& \alpha^{\prime}{ }_{12}=q_{1 j} c_{j 1} P_{j 2}+q_{1 j} c_{j 2}
\end{aligned}
$$

where $P_{j 1}$ is the probability that the $1 / 2-1 / 2$ photon has survived $n$ absorptions and $\mathrm{P}_{\mathrm{j} 2}$ is the probability that the $1 / 2$ - $1 / 2$ photon has been converted to a $3 / 2-1 / 2$ photon in $n$ or less absorptions. The probability of the excited ion giving a $1 / 2-1 / 2$ photon after each absorption is $C_{j 1}(\approx .96)$ and a $3 / 2-1 / 2$ photon is $C_{j 2}(\approx .04)$. Calculations show that $\alpha^{\prime}{ }_{11} / \alpha^{\prime}{ }_{12}$ can be reduced to the observed ratio of line strengths $(\approx 2.0)$ after about 10 absorptions of the $1 / 2-1 / 2$ photons (the corona is assumed optically thin for the $3 / 2-1 / 2$ photon).

The expected number of absorptions of a $1 / 2-1 / 2$ photon in traveling a distance of one scale height can be estimated following the method of Ivanov-Kholodnyi and Nikol'skii (1961). They give the total time that a photon is in an absorbed state, while traveling over a certain distance, by the expression

$$
t \approx \tau^{2} / \mathrm{A}
$$

Where $\tau$ is the optical depth and $\mathrm{A}$ is the spontaneous transition 
- probability. Now the number of times a photon is absorbed in traveling a certain distance is

$$
\mathrm{n} \approx t / \mathrm{T}_{\mathrm{a}}=\mathrm{t} \cdot \mathrm{A}=\mathrm{T}^{2}
$$

where $\mathrm{T}_{\mathrm{a}}$ is the lifetime of each absorption. The optical depth for the $1 / 2-1 / 2$ photon is in the range $2-20$, taking the product of electron density and scale height as $10^{19}-10^{20}$. It is thus seen that $n \approx 10$ is a possible result for the corona. At this stage it is difficult to say definitely if this mechanism will give the desired re-distribution of the line strengths, but it seems worth further investigation. Also, if effective, this mechanism may influence the excitation of the $\lambda 5303$ coronal line.

I am grateful to $W$. Neupert and $E$. Smith for the relative line strengths prior to publication, to $S$. Kastner for helpful discussions and to D. Osterbrock for several discussion, especially those concerning the exchange cross-sections and the self-absorption mechanism. 


\section{REFERENCES}

1. Garstang, R.H., 1962, Ann. d'Ast., 25, 109.

2. Ivanov-Kholodnyi, G. S. and Nikol'skii, G. M., 1961. Soviet Astronomy $\underline{5}, 31$.

3. Neupert, W. M., and Smith, E. V. P., 1964, Paper given at June 24-27 meeting of the American Astronomical Society.

4. Osterbrock, D. E., 1963, Planet. Space Sci., 11, 621 .

5. Seaton, M. J. 1958, Rev. Mod. Phys., 30, 979.

6. 1963, Planet. Space Sci., 12, 55.

7. St. John, R. M., Miller, F. L. and Lin, C. C. 1964, Phys. Rev., 134, A888.

8. Van Regemorter, H. 1962, Ap. J., 136, 906. 